\title{
CICLO DE VIDA PÓS-MARSUPIAL E CRESCIMENTO DE METAMYSIDOPSIS ELONGATA ATLANTICA (CRUSTACEA, MYSIDACEA, MYSIDAE) EM CULTIVO DE LABORATÓRIO
}

\author{
Andréa Mara da Silva Gama ${ }^{1}$ \\ Mônica Adelina Montú ${ }^{1}$ \\ Luiz Felipe M. de Gusmão ${ }^{1}$
}

\begin{abstract}
PÓS-MARSUPIAL LIFE CICLE AND GROWTH OF METAMYSIDOPSIS ELONGATA ATLANTICA (CRUSTACEA, MYSIDACEA, MYSIDAE) UNDER LABORATORY CONDITION. Metamysidopsis elongata atlantica (Bacescu, 1968) was reared in the laboratory for 45 days at $20 \pm 1{ }^{\circ} \mathrm{C}$ and salinity of 30ppt. Growth curves (von Bertalanffy model) were calculated for both sexes and for each sex. The daily rate of carapace growth was significantly different between females and males ( $F$ test, $p$ $<0.05$ ). Before the sexual maturity (14 days), the growth rate of females was higher than that of males (females, $0.0457 \mathrm{~mm} \mathrm{day}^{-1}$; males, $0.0448 \mathrm{~mm} \mathrm{day}^{-1}$ ). After the maturity (15 to 45 days), these rates decreased similarly for both sexes (females, $0.0203 \mathrm{~mm}^{-1 a y}{ }^{-1}$; males, $0.0174 \mathrm{~mm} \mathrm{day}^{-1}$ ). The average growth rate was $0.0207 \mathrm{~mm}_{\text {day }}{ }^{-1}$ over the 45 days. Twelve molts were observed in a period of 60 days. The first five molts occurred up to 14 days old (age of the sexual differentiation), with a mean intermolt period of 2.9 days. From the $6^{\text {th }}$ molt it increased to 5.6 days. The results suggest that the use of the carapace length is a good measure to calculate the growth and longevity of the organisms.
\end{abstract}

KEYWORDS. Crustacea, Mysidae, Metamysidopsis, growth, molts.

\section{INTRODUÇÃO}

Metamysidopsis elongata atlantica (Bacescu,1968) é freqüente e abundante em zonas neríticas do oceano Atlântico, distribuindo-se livremente em grandes manchas na área de arrebentação (MAUChLINE, 1980). No Brasil, a espécie foi registrada no litoral de Cananéia, São Paulo (AlmeIDA-Prado, 1974), em forma de densas populações na zona de arrebentação das praias arenosas do litoral sul do Rio Grande do Sul (GAmA \& ZAMBONI, 1999). Os Mysidacea têm reprodução sexuada e a cópula ocorre geralmente durante a noite, com duração de poucos minutos. Os óvulos são fertilizados imediatamente após a expulsão dos ovidutos, sendo introduzidos no marsúpio, onde ocorre o desenvolvimento larval (MAUCHLINE, 1980). Os jovens emergem do marsúpio em estágio juvenil de desenvolvimento semelhante

1. Fundação Universidade Federal do Rio Grande (FURG), Depto de Oceanografia, Lab. de Zooplâncton, C.P. 474, 96.201-900, Rio Grande, RS, Brasil.(docamsg@super.furg.br; docmontu@super.furg.br; ocealfmg@super.furg.br) 
ao adulto. Como ocorre nos crustáceos em geral, devido à presença de exoesqueleto, o crescimento morfológico do animal é descrito pelas suas mudas (CLUTTER \& THEILACKER, 1971).

MAUCHLINE $(1976,1977)$ mostrou que o crescimento de alguns isópodos, eufausiáceos e crustáceos decápodes pode ser descrito em termos de períodos de intermudas e fatores de crescimento. Um período de intermuda compreende duas mudas sucessivas, e o fator de crescimento é a porcentagem de aumento de tamanho em comprimento entre cada muda. $\mathrm{O}$ período de intermuda e o fator de crescimento foram descritos como uma função exponencial do comprimento do animal ou número de mudas. AsTTHORSSON \& RALPH (1984) descreveram para Neomysis integer (Leach, 1814) que a duração do período de intermudas depende do comprimento do espécime e da temperatura em que eles crescem.

Considerando o potencial e a importância dos Mysidacea, tanto como componentes essenciais nas cadeias alimentares marinhas e estuarinas (MARKLE \& GRANT, 1970), como seu uso freqüente em testes de toxicidade devido à sua sensibilidade a baixas concentrações de substâncias tóxicas (NIMMO \& HAMAKER, 1982), autores têm estudado seu crescimento e desenvolvimento em laboratório. Metamysidopsis elongata (Holmes, 1900) da costa do Pacífico, Leptomysis lingvura Sars, 1869 e Hemimysis speluncola (Ledoyer, 1963) das costas da Europa (Mar Mediterrâneo e Mar do Norte) tiveram suas curvas de crescimento estudadas em profundidade (ClutTer \& TheIlacker, 1971; GAudy \& Guérin, 1979). Alguns aspectos do crescimento, oriundos de populações cultivadas em laboratórios, foram abordados para Leptomysis lingvura, Neomysis integer, N. mercedis Holmes, 1897 e Mysidopsis almyra Bowman, 1964 (WittMann, 1981; Bremer \& ViJverberg, 1982; AstTHORSSOn \& RalPh 1984; Brandt et al., 1993; Domingues et al., 1999).

O objetivo foi estudar o crescimento dos indivíduos e analisar o ciclo de mudas de Metamysidopsis elongata atlantica, da região sul do Brasil e cultivados em laboratório, com temperatura e salinidade controladas.

\section{MATERIAL E MÉTODOS}

Foram retiradas aleatoriamente, dos tanques de cultivo massivo, fêmeas de M. elongata atlantica com marsúpio visivelmente distendido e esbranquiçado e colocadas separadamente em recipientes com água do mar. A alimentação consistiu em 100 náuplios de Artemia/organismo/dia, até a desova. Após a desova, cada um dos 101 juvenis eclodidos foi isolado em recipiente de polietileno contendo $200 \mathrm{ml}$ de água do mar filtrada, em condições semelhantes às do cultivo das matrizes. Os animais foram mantidos durante os 60 dias da realização do teste em condições de fotoperíodo $12: 12 \mathrm{~h}$, temperatura de $20 \pm 1^{\circ} \mathrm{C}$, salinidade de $30 \pm 1 \%$ e luminosidade de 300 Lux. Os juvenis foram alimentados diariamente com náuplios de Artemia sp. nas seguintes quantidades: de 1 a 5 dias, 15 náuplios Artemia/dia; de 6 a 10 dias, 30 náuplios Artemia/dia; de 11 a 20 dias, 50 náuplios Artemia/dia e, após 20 dias de idade, 100 náuplios Artemia/dia (GAmA \& Zamboni, 1999).

As exúvias foram verificadas diariamente a partir da eclosão dos jovens, até o final do experimento; quando presentes, foram removidas e acondicionadas em tubos de Eppendorf contendo formol $4 \%$, tamponado com borato de sódio, para posterior medição em microscópio estereoscópico. As medidas foram efetuadas entre o ápice do rostro e o extremo posterior da carapaça.

O modelo de BERTALANFFy (1938) foi utilizado para expressar o aumento em comprimento da carapaça, sendo a equação geral descrita como: $\mathrm{Lt}=\mathrm{L} \infty\left[1-\mathrm{e}^{-\mathrm{k}(\mathrm{t}+\mathrm{t} 0)}\right]$ onde; $\mathrm{Lt}=$ comprimento estimado relativo à idade $\mathrm{t} ; \mathrm{L} \infty=$ comprimento teórico (assintótico); $\mathrm{e}=$ base dos logaritmos neperianos; $\mathrm{k}=$ constante de catabolismo (anual); $\mathrm{t}=$ idade considerada (dias); $\mathrm{t} 0=$ idade inicial ou hipotética onde o indivíduo tem comprimento igual a zero. As medidas de comprimento da carapaça foram relacionadas com idades (dias) e os parâmetros da curva estimados através de ajuste não linear. Nesta etapa, fez-se uso da ferramenta "Solver" do software Microsoft Excel, que realiza a minimização da soma do quadrado dos resíduos, estimando novos parâmetros para o modelo de Bertalanffy a partir de "valores sementes". 
A aderência das curvas selecionadas foi testada através de correlação entre os pontos observados e a curva estimada, aceitando-se valores de coeficiente " $r$ " acima de 0,90 ; realizou-se a comparação estatística entre as curvas pelo método da verossimilhança (CERRATO, 1990).

\section{RESULTADOS}

As equações obtidas para o ajuste das curvas de crescimento da carapaça de fêmeas e machos e para ambos os sexos foram: fêmeas (fig. 1) $\mathrm{L}_{\mathrm{t}}=2,05\left[1-\mathrm{e}^{-0,0397(\mathrm{t}+7,03226)}\right] ; \mathrm{R}=$ 0,98; Longevidade $=116,13$ dias. Machos (fig. 2) $\mathrm{L}_{\mathrm{t}}=1,86\left[1-\mathrm{e}^{-0,04651(\mathrm{t}+6,74613)}\right] ; \mathrm{R}=0,98$; Longevidade $=99,02$ dias. Machos e fêmeas (fig. 3) $\mathrm{L}_{\mathrm{t}}=1,943\left[1-\mathrm{e}^{-0,0435(\mathrm{t}+6,81084)}\right] ; \mathrm{R}=$ 0,97 ; Longevidade $=105,81$ dias. As curvas de crescimento para fêmeas e machos são significativamente diferentes (Teste $\mathrm{F}, \mathrm{p}<0,05$ ).

A taxa de crescimento da carapaça calculada para fêmeas de 0-14 dias, foi de $0,0457 \mathrm{~mm} /$ dia e do $15^{\circ}$ ao $45^{\circ}$ dia, $0,0203 \mathrm{~mm} /$ dia (fig. 1). Para machos de $0-14$ dias foi de $0,0448 \mathrm{~mm} /$ dia e do $15^{\circ}$ ao $45^{\circ}$ dia, de $0,0174 \mathrm{~mm} /$ dia (fig. 2). A taxa de crescimento da carapaça calculada para todos os indivíduos, da eclosão ao $14^{\circ}$ dia, foi de $0,0453 \mathrm{~mm} / \mathrm{dia}$ e do $15^{\circ}$ ao $45^{\circ}$ dia de $0,0187 \mathrm{~mm} /$ dia. A taxa de crescimento total da carapaça, em 45 dias, foi de $0,0270 \mathrm{~mm} /$ dia (fig 3 ).

Em 60 dias, Metamysidopsis elongata atlantica alcançou o total de 12 mudas, tanto para machos como para fêmeas (fig. 4). Observou-se que apenas um macho fez a $13^{\mathrm{a}}$ muda. Até a maturação sexual (14 dias), ocorreram 5 mudas em intervalos regulares; posteriormente tornaram-se dessincronizadas e com menor freqüência (fig. 4). No $3^{\circ}, 6^{\circ}, 8^{\circ}, 10^{\circ}$ e $14^{\circ}$ dias de cultivo ocorreram, respectivamente a $1^{\mathrm{a}}, 2^{\mathrm{a}}, 3^{\mathrm{a}}, 4^{\mathrm{a}}$ e $5^{\mathrm{a}}$ muda, para machos e fêmeas. $\mathrm{O}$ período médio de intermudas pós-marsupiais foi de 2,9 dias em um ciclo de 14 dias, tanto para machos quanto para fêmeas, aumentando para 5,4 dias nas fêmeas e 5,8 dias nos machos, do $15^{\mathrm{o}}$ ao $60^{\mathrm{o}}$ dia (figs. 5, 6). Para ambos os sexos, Metamysidopsis elongata atlantica apresentou um período médio de intermuda de 2,9 dias até sua diferenciação sexual (14 dias), e de 5,6 dias do $15^{\circ}$ ao $60^{\circ}$ dia (fig. 7). A partir do $25^{\circ}$ dia; constatou-se fêmeas ovadas nos recipientes de cultivo, com uma tendência das mudas ocorrerem antes da formação dos ovos no marsúpio das fêmeas (fig. 4).

\section{DISCUSSÃO}

Poucos autores citam a existência de mudas intramarsupiais com diferentes espécies de Mysidacea. NAIR (1939), por meio de observações diretas, descreveu para Mesopodopsis orientalis (Tattersall, 1908) três estágios larvais marsupiais, com as três mudas ocorrendo dentro do marsúpio. ClutTER \& THeILACKer (1971) descreveram para Metamysidopsis elongata a ocorrência de uma muda no interior do marsúpio, em temperaturas entre $14 \mathrm{e}$ $20^{\circ} \mathrm{C}$, sem especificar valores de salinidade, mas relatando que o cultivo foi realizado em água do mar. Trabalharam com organismos procedentes da Califórnia, no Pacífico, e seus resultados diferem dos apresentados neste trabalho. Em cultivo, eles observaram 10 mudas para fêmeas e machos, sem especificar se as mudas intramarsupiais foram somadas às pósmarsupiais, ou se foram computadas somente as pós-marsupiais. A temperatura de cultivo utilizada por CLUTTER \& THEILACKER (1971), oscilou entre $14^{\circ}$ e $20^{\circ} \mathrm{C}$, seguindo as variações do ambiente natural; não foi mencionada a salinidade utilizada no cultivo. A temperatura e 

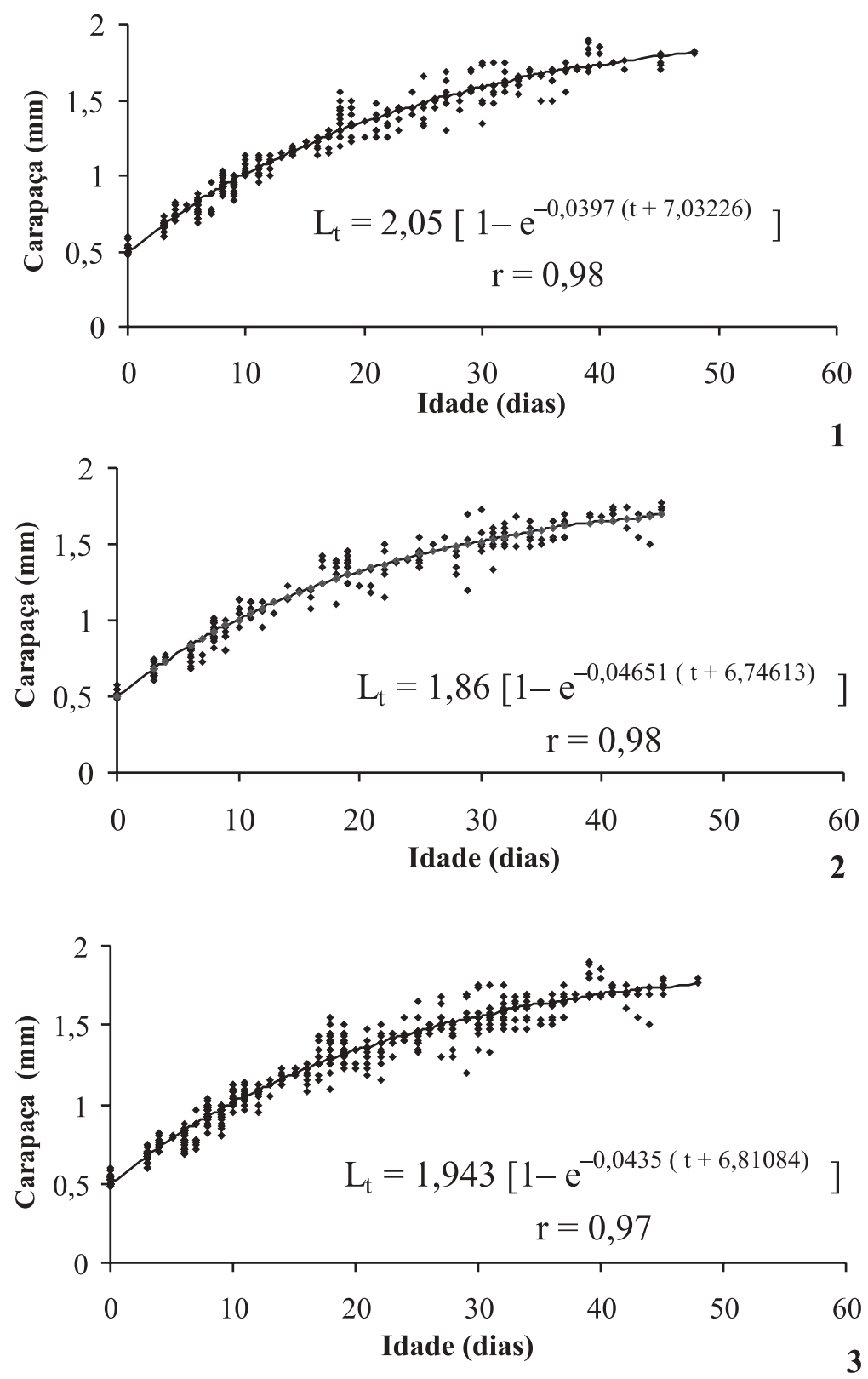

Figs. 1-3. Curva de crescimento de Metamysidopsis elongata atlantica em cultivos à temperatura de $20 \pm 1{ }^{\circ} \mathrm{C} \mathrm{e}$ salinidade $30 \pm 1$ : 1 , fêmeas; 2 , machos; 3 , machos e fêmeas. 
salinidade dos cultivos aqui obtidos foram mantidas constantes $\left(20^{\circ} \pm 1 \mathrm{C}\right.$ e $30 \pm 1 \%$ ), verificando-se um total de 12 mudas, em 60 dias. Observaram-se 5 mudas pós-marsupiais para ambos os sexos, coincidindo com o resultado de NAIR (1939).

Os períodos de intermudas ora obtidos foram semelhantes até a maturação sexual, tanto para machos como para fêmeas. Comparando com os resultados registrados por ClutTer \& THEILACKer (1971), que encontraram períodos de intermudas variando entre 5 e 13 dias (média de 10 dias), pode-se observar que a ampla variação de temperatura utilizada por estes autores pode ter influenciado os resultados, tanto nos períodos de intermuda como na duração dos estágios. Durante a realização dos presentes testes, a temperatura foi mantida praticamente constante, com variação de $\pm 1^{\circ} \mathrm{C}$, o que permitiu um desenvolvimento mais regular dos organismos. Os resultados encontrados para $M$. elongata atlantica foram bastante semelhantes aos que Loureiro Fernandes \& GAMA (1996) descreveram para Metamysidopsis munda (Zimmer, 1918), em que as mudas ocorreram em intervalos regulares (2,2 e 2,7 dias) até a maturação sexual, obtida após a $5^{\mathbf{a}}$ muda, em temperatura de $20^{\circ} \mathrm{C}$ e salinidade de $30 \%$. Esta equivalência de resultados, provavelmente evidencie que as duas espécies apresentam semelhanças acentuadas no ritmo de mudas e crescimento por ocorrerem na mesma área e época do ano, assim como por serem pertencentes ao mesmo gênero. Lussier et al. (1988) encontraram para Mysidopsis bahia Molenock, 1969 , cultivada a $25^{\circ} \mathrm{C}$ e salinidade de $30 \%$, 9 mudas nos 18 primeiros dias de vida, com períodos de intermuda de 2,3 dias.

Em M. elongata atlantica constatou-se uma tendência das mudas ocorrerem imediatamente antes do aparecimento de ovos não-fecundados no marsúpio das fêmeas, aos 25 dias. Nesta mesma espécie, Ademilson J. Zamboni (com. pess.) verificou ovos nãofecundados aos 14 dias, mas as fêmeas estavam junto aos machos, o que poderia ter influência na aceleração da desova. Após a liberação dos juvenis, observou-se mudas nos recipientes de manutenção das fêmeas, indicando que o processo de mudas nas fêmeas adultas de M. elongata atlantica e a eclosão dos jovens ocorrem simultaneamente. Essa observação é semelhante à constatada para Leptomysis lingvura (WITTMANN, 1981).

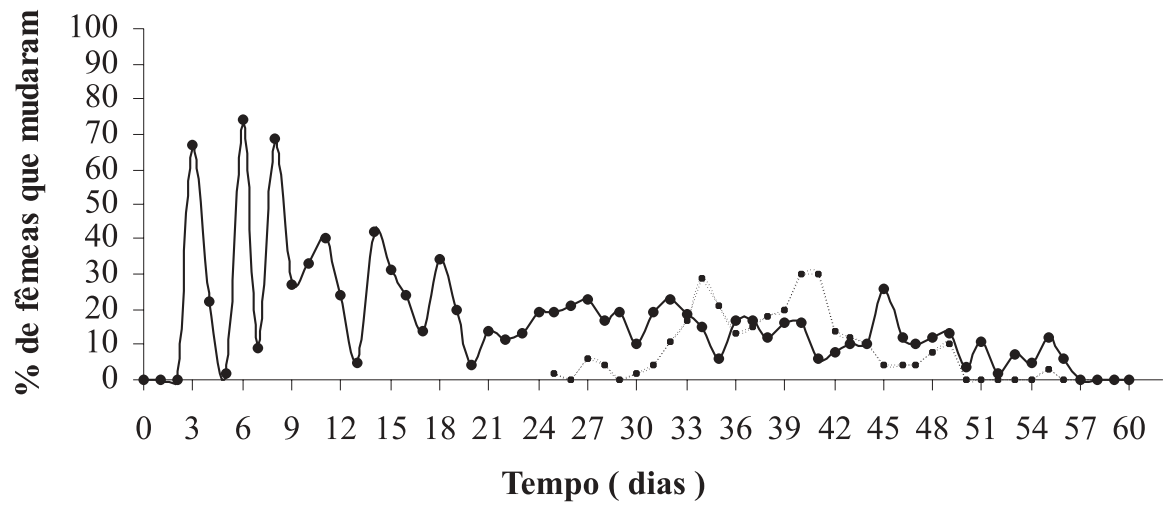

Fig. 4. Ciclo de vida de fêmeas de Metamysidopsis elongata atlantica em cultivos à $20 \pm 1^{\circ} \mathrm{C}(\cdot$ mudas; --- período de reprodução). 

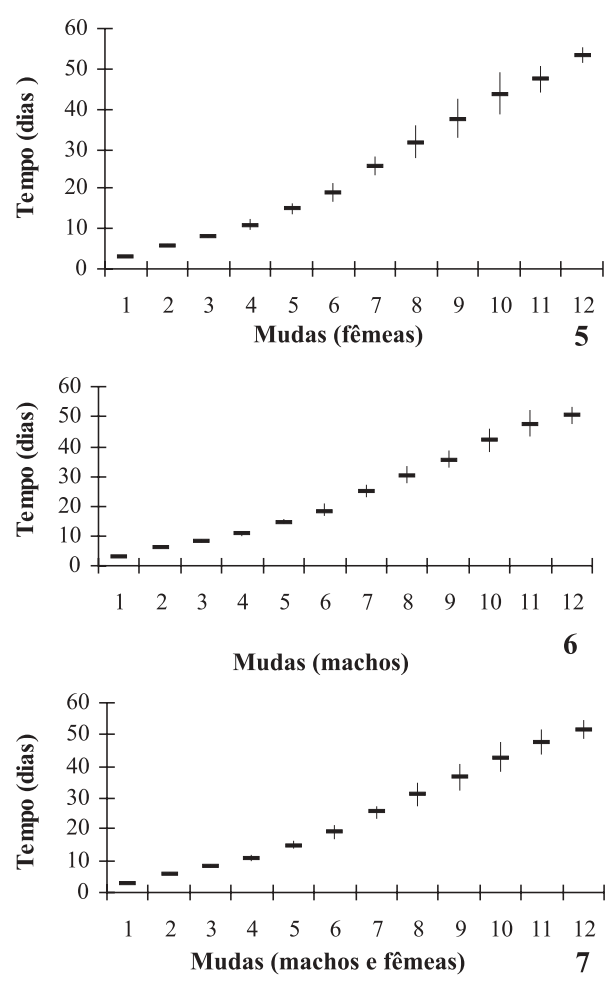

Figs. 5-7. Freqüência de mudas de Metamysidopsis elongata atlantica em cultivos à $20 \pm 1^{\circ} \mathrm{C}$ e salinidade de $30 \pm$ 1: 5 , fêmeas ; 6 , machos; 7 , machos e fêmeas ( - , média; $\mid, \pm$ desvio padrão).

Clutter \& Theilacker (1971) citaram que as fêmeas adultas do gênero Metamysidopsis mudam antes de serem fecundadas, logo após a liberação de suas ninhadas, fato ora confirmado. Para M. elongata atlantica, as mudas foram sincronizadas até o desenvolvimento da principal característica sexual secundária (aparecimento do marsúpio) no $14^{\circ}$ dia, ocorrendo a intervalos regulares de 2,9 dias, passando depois a intervalos maiores (5,6 dias), após a maturação, concordando com CLUTTER \& THEILACKER (1971) e Lussier et al. (1988).

Os dados aqui obtidos discordam, em parte, dos encontrados por ClutTer \& THEILACKer (1971) para Metamysidopsis elongata, do Pacífico, para o qual o crescimento e taxas de mudas foram diferentes para machos e fêmeas. Em relação às taxas de crescimento, estes autores não fornecem informações, impedindo comparações com as espécies do Atlântico. A taxa de mudas apresentou-se igual tanto para machos quanto para fêmeas, ocorrendo 12 mudas em 60 dias. A taxa de crescimento diário da carapaça foi diferente entre os sexos, sendo maior nas fêmeas $(0,0457 \mathrm{~mm} / \mathrm{dia})$ do que nos machos $(0,0448 \mathrm{~mm} /$ dia) antes da maturidade sexual (14 dias), e decrescendo tanto para fêmeas $(0,0203 \mathrm{~mm} /$ dias) quanto para machos $(0,0174 \mathrm{~mm} /$ dia $)$ após a maturidade. $O$ crescimento médio diário ao longo de 45 dias para todos os organismos foi de $0,0207 \mathrm{~mm} / \mathrm{dia}$. 
Os resultados obtidos permitem sugerir o uso do comprimento da carapaça como adequado para avaliação do crescimento e determinação da curva para Mysidacea, sem necessidade de outras medidas, minimizando o manuseio, reduzindo o estresse do organismo cultivado e possibilitando ainda diferenciar os estágios larvais.

Agradecimentos. À FAPERGS (Processo 98/0739. 4) e ao CNPq (Processos 52.1534/96 e 52.1537/96/ 7) pelo apoio financeiro. À Fundação Universidade Federal do Rio Grande pelo apoio para a realização das coletas, aos técnicos e docentes do Laboratório de Crustáceos Decápodes, e ao Dr. Fernando D’Incao pelas sugestões e auxílio.

\section{REFERÊNCIAS BIBLIOGRÁFICAS}

Almeida-Prado, M. S. 1974. Sistemática dos Mysidacea (Crustacea) na região de Cananéia. Bolm Inst. Oceanogr. São Paulo, São Paulo, 23:47-87.

AstTHORSSON, O. S. \& RalPh, R. 1984. Growth and moulting of Neomysis integer (Crustacea: Mysidacea). Mar. Biol., Berlin, 79:55-61.

Bertalanffy, L. 1938. A quantitative theory of growth. Hum. Biol., Baltimore, 10(2):181-213.

Brandt, O. P. M.; Fujimura, R. W. \& Finlayson, B. J. 1993. Use of Neomysis mercedis (Crustacea: Mysidacea) for estuarine toxicity tests. Trans. Am. Fish. Soc., St. Paul, 122:279-288.

Bremer, P. \& Vijverberg, J. 1982. Production, population biology and diet of Neomysis integer (Leach) in a shallow Frisian lake (The Netherlands). Hydrobiologia, Dordrecht, 93:41-51.

Cerrato, R. M. 1990. Interpretable statistical tests for growth comparisons using parameters in the von Bertalanffy equation. Can. J. Fish. aquat. sci., Ottawa, 47:1416-1426.

Clutter, R. I. \& TheILACKer, G. H. 1971. Ecological efficiency of a pelagic mysis shrimp: estimates of growth and mortality studies. Fish. Bull., Sacramento, 69:93-115.

Domingues, P. M.; Turk, P. E. et al. 1999. Culture of the mysid, Mysidopsis almira (Bowman), (Crustacea: Mysidacea) in a static water system: effects of density and temperature on production, survival an growth. Aquacult. Research, Oxford, 30:135-143.

Gama, A. M. S. \& Zamboni, A. J. 1999. Aspectos da biologia e do cultivo de Metamysidopsis elongata atlantica para uso em testes de toxicidade. Nauplius, Rio Grande, 7:127-139.

GAUdy, R. \& GuÉRIN, J. P. 1979. Ecophysiologie compareé des mysidaces Hemimysis speluncola Ledoyer (cavernicole) et Leptomysis lingvura G. O. Sars (non cavernicole). Action de la température sur la croissance em élevage. J. exp. mar. Biol. Ecol., Amsterdam, 38:101-109.

Loureiro Fernandes, L. \& Gama, A. M. S. 1996. Molting cycle in Metamysidopsis munda (Crustacea: Mysidacea). Nauplius, Rio Grande, 4:171,172.

Lussier, S. M.; KHUN, A. et al. 1988. Techniques for the laboratory culture of Mysidopsis species. Environ. Toxicol. Chem., Houston, 7:969-977.

MarkLE, D. F. \& GRANT G. C. 1970. The summer food habits of young-of-the-year striped bass in three Virginia rivers. Chesap. Sci., Solomons, 11:50-54.

Mauchline, J. 1976. The Hiatt growth for diagram for Crustacea. Mar. Biol., Berlin, 35:79-84.

- 1977. Growth of shrimps, crabs and lobster - an assessment. J. Cons. perm. int. Explor. Mar., Copenhagen, 37:162-169.

1980. The biology of mysids and euphausiids. Adv. Mar. Biol., London, 18:1-369.

NAIR, K. B. 1939. The reproduction, oogenesis and development of Mesopodopsis orientalis Tatt. J. exp. mar. Biol. Ecol., Sect. B, Amsterdam, 9(4):175-223.

Nimmo, D. R. \& Hamaker, T. L. 1982. Mysids in toxicity testing - a review. Hydrobiologia, Dordrecht, 93:171178.

WittMann, K. J. 1981. On the breeding biology and physiology of marsupial development in Mediterranean Leptomysis (Crustacea: Mysidacea) with special reference to the effects of temperature and size eggs. J. Exp. Mar. Biol. Ecol., Amsterdam, 53:261-279.

Recebido em 09.02.2001; aceito em 19.02.2002 